\title{
American Exceptionalism: Determinants of spreading COVID-19 misinformation online in five countries
}

\author{
Mark Pickup \\ Simon Fraser University \\ mark_pickup@sfu.ca
}

\author{
Dominik A. Stecula \\ Colorado State University \\ dominik.stecula@colostate.edu cliff.vanderlinden@mcmaster.ca
}

This is a pre-print. This manuscript has not been peer-reviewed.

Version 1.0 


\begin{abstract}
Social media have long been considered a venue in which conspiracy theories originate and spread. It has been no different during COVID-19. However, understanding who spreads conspiracy theories by sharing them on social media, and why, has been underexplored, especially in a cross-national context. The global nature of the novel coronavirus pandemic presents a unique opportunity to understand the exposure and sharing of the same COVID-19 related conspiracies across multiple countries. We rely on large, nationally representative surveys conducted in July of 2020 in the United States, United Kingdom, Canada, Australia, and New Zealand, to begin to understand who shares conspiracies on social media and what motivates them. We find that Americans are no more likely to encounter prominent COVID-19 conspiracies on social media but are considerably more likely to subsequently share them. In all countries, trust in information from social media predicts conspiracy theory sharing, while in the US politics plays a unique role Our results make clear that American behavior on social media has the potential to poison online public discourse globally.
\end{abstract}

\title{
Main
}

Since the emergence of the novel coronavirus pandemic, social scientists have been working on identifying causes and correlates of COVID-19 related attitudes and behaviors. A lot of that focus has been centered on COVID-19 misinformation, and specifically endorsement of different coronavirus related conspiracy theories. These conspiracies matter, because belief in them has been found to have implications for public health behavior, such as compliance with mask wearing or social distancing, or willingness to accept a COVID-19 vaccine when it becomes available. Much of this research has focused on social media, as the environment in which these theories originate and proliferate ${ }^{1,2}$. Existing research, however, does not really shed light onto who is sharing and proliferating these conspiracies on social media, nor does it explore these behaviors across different national contexts. In fact, the bulk of the existing work has focused on the United States. In this article, we examine individual level predictors of sharing, and therefore spreading, COVID-19 conspiracies online based on the results of five large, nationally representative surveys fielded during the pandemic (July of 2020) in the United States, Canada, United Kingdom, Australia, and New Zealand.

We find that seeing conspiracy theories online and trusting that social media provides factual and objective information on COVID-19 are important predictors of sharing conspiracies. This is true across all five countries in our study, but the US stands out in terms of the extent to which seeing a conspiracy translates into sharing it. We also find that the US is distinct in the degree to which political factors such as populism and ideology predict the sharing of conspiracies online. This is consistent with evidence that COVID-19 is more politicized in the US compared to other nations. Finally, we find some evidence that in countries in which political considerations are not a determinant, trust in health officials is a predictor of sharing conspiracies. Trust that 
health officials provide factual and objective information reduces such sharing of conspiracies.

The emergence of the global pandemic in 2020 presented a ripe opportunity for the spread of a variety of conspiracy theories pertaining to the origins of the pandemic, the severity of COVID-19, and the ways of dealing with it. At the beginning of the pandemic, a handful of conspiracies about the virus escaping from a Wuhan lab, or that it is a bioweapon circulated heavily ${ }^{3}$. Over time, however, an increasing amount of conspiracies surrounding COVID-19 began to emerge. Beyond just being inaccurate, these beliefs have real world consequences. Scholars have demonstrated that those believing in COVID-19 conspiracies were less likely to engage in social distancing, increased handwashing, mask wearing, and other public health recommended behaviors ${ }^{4,5,6,7}$. Additionally, people holding these beliefs are more likely to reject the COVID-19 vaccine, which is consistent with general work on conspiracy theories and vaccine intention ${ }^{1,5,8,9,10}$. Finally, they might have consequences beyond the realm of public health, as these beliefs might also fuel racially-charged attacks against Asian Americans ${ }^{2,11}$.

Social media have been identified as one of the key venues in which conspiracy theories originate and spread $^{2,12}$. Previous work in the context of the COVID-19 pandemic shows an important role that the information environment plays on being misinformed about COVID-19 and believing in various conspiracy theories. Those who rely on social media for information have been found to be more misinformed about basic facts surrounding the novel coronavirus and endorse various conspiracies related to $i^{4,13,14}$. Researchers have also found that these conspiracies then frequently enter the mainstream, amplified by traditional media outlets ${ }^{2}$. For example, in the early days of the pandemic, conspiracy theories about coronavirus being a bioweapon or escaping from a lab in Wuhan were amplified by conservative news media in the United States ${ }^{3}$.

In this paper, we focus on the viewing and sharing of the following seven conspiracy theories:

- The Chinese government engineered the coronavirus in a lab.

- The pharmaceutical industry is involved in the spread of the coronavirus.

- There is a link between $5 \mathrm{G}$ technology and the coronavirus.

- The United States military developed the coronavirus as a bioweapon.

- Bill Gates is using the coronavirus to push a vaccine with a microchip capable of tracking people.

- The coronavirus escaped from a lab in Wuhan.

- The worldwide death toll from COVID-19 is highly exaggerated.

These are, by no means, all of the COVID-19 conspiracies. Given the global nature of the crisis and the impact COVID-19 has had on the lives of nearly everyone on the 
planet, the amount of different theories, or the different versions of the above theories has been staggering. But these theories have been some of the most popular forms of misinformation surrounding COVID-1915. Furthermore, these claims originated on obscure websites or on nebulous social media profiles, and were proliferated by other social media accounts"15,16. For example, the claim that "Bill Gates is using the coronavirus to push a vaccine with a microchip capable of tracking people" has its origins on an obscure website BioHackInfo.com, and it was then amplified by a YouTube video from the Jacksonville, Florida-based Law of Liberty Baptist Church ${ }^{15}$.

Thanks to the research highlighted above, we know that conspiracy theory beliefs matter, and that these theories tend to spread on social media. What we know much less about is who exactly spreads these theories by sharing them online and why do they engage in such behavior. This is the motivating question of our work. Based on existing research, we derive several expectations for who will share COVID-19 conspiracies on their social network accounts. First, we expect that those who report seeing conspiracy theories on social media will be more likely to share them. As the work of Van Bavel and co-authors ${ }^{17}$ shows, one does not have to believe in conspiracy theories to share them online, but being exposed to these theories is of crucial importance.

We also expect that trust is an important determinant of sharing. For starters, conspiracy theories generally spread expeditiously under the conditions of high uncertainty and low trust in official information sources ${ }^{2}$. Previous work also highlights the importance of trust in health officials to acceptance of common vaccine-related misinformation ${ }^{6,18}$. As a result, we expect to see those who trust that the information from health officials as factual and objective to be less likely to share conspiracy theories. On the flip side, we expect those who trust information coming from social media to engage in more sharing of these conspiracies ${ }^{19}$.

We also expect politics to play a big role as a determinant of spreading conspiracies online, at both the individual and country level. First, previous work has shown that, especially during a pandemic, populist attitudes are an important determinant of conspiracy theory endorsement ${ }^{7}$. That is because some of the key components of populist attitudes include anti-elitism and anti-intellectualism, which play a big role in rejecting public health information from experts. Furthermore, because populism can thrive across the traditional left-right political spectrum, it is an important independent driver of conspiracy theory endorsement, above and beyond partisanship or ideology ${ }^{7}$. As a result, we expect those high in populism to be more likely to share conspiracy theories on social media than those low in populism.

Secondly, ideology might play a role in sharing COVID-related conspiracy theories on social media. In general, there is not a clear relationship between left-right ideology 
and belief in conspiracy theories. A lot depends on the conspiracy theory itself, and who it identifies as a culprit and the victims ${ }^{20}$. At the same time, in the context of the COVID-19 pandemic specifically, researchers have discovered that, in general, conservatives have been more likely to endorse COVID-19 conspiracies, including

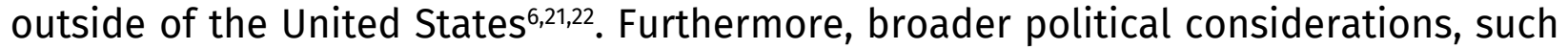
as ideology, have been found to be associated with a variety of COVID-related attitudes and behaviors, in both the US and elsewhere ${ }^{23,24,25}$. Additionally, behavioral evidence of sharing misinformation during the 2016 Presidential election in the US suggests that conservatives were much more likely to share fake news than liberals, though part of that pattern is likely due to the fact that most fake news in that election cycle was targeted to conservatives ${ }^{26}$. As a result, we expect left-right ideology to play a role in sharing conspiracy theories on social media, with conservatives (those identifying as political right) being more likely to share them than liberals (those identifying as political left).

For the same reason that we expect ideology to play a role, trust in government may also be important. Part of this is because a general distrust of government is an important element of populism. Beyond that, trust in government is likely driven by partisanship. Those that identify with the party in government are more likely to trust the government and the information coming from the government. Meanwhile, those that did not identify with the party in government are more likely to distrust the government and the information from it. To the extent that a government engages with COVID-related conspiracies, this partisan motivated trust could have consequences for whether an individual shares conspiracy related information.

This highlights the importance of the political context of COVID-19 conspiracy information. As scholars have noted, the pandemic has been exceptionally politicized in some parts of the world ${ }^{27}$. During the early days of the pandemic, President Donald Trump, and his allies in the conservative media downplayed the danger of the novel coronavirus and accused other news sources and Democratic politicians of creating mass hysteria and panic. The tone of coverage changed after President Trump declared a national emergency on March 13, 2020, but the broader point is that from the very beginning, politicians across the political spectrum in the US were sending very different signals about the nature of COVID-19,28. That politicization was not the case in a lot of other countries. In Canada, for example, there was (mostly) a consensus among political elites about the coronavirus during the early stages of COVID-19 pandemic ${ }^{29}$.

To highlight just how politicized COVID-19 has been in the US compared to the other countries in our study, we have gathered original data from the MediaCloud Explorer database, following the work of Motta and coauthors ${ }^{3}$. We calculated how politicized national news media coverage of COVID-19 is in each country from January 1, 2020 to July 1,2020 , which is a period of time preceding when our surveys were fielded (details on how we identified politicized media coverage is available in the Methods section). Consistent with expectations, we find that national news media is most politicized in 
the United States (26\%, or more than a quarter of all COVID-19 coverage, is politicized in the US, compared to only $13 \%$ in Canada). We also find that in terms of volume, the sheer amount of politicized news coverage in the US vastly exceeds the volume of politicized coverage in other countries. In fact, there are considerably more politicized stories in the national US news media than in the other four countries combined. We expect political considerations to matter the most in countries where COVID-19 has been the most politicized. As a result, we expect to see a bigger role for politics (ideology, populism and trust in government information) in the United States than in the other countries.

Figure 1. Politicization of COVID-19 News Coverage by Country

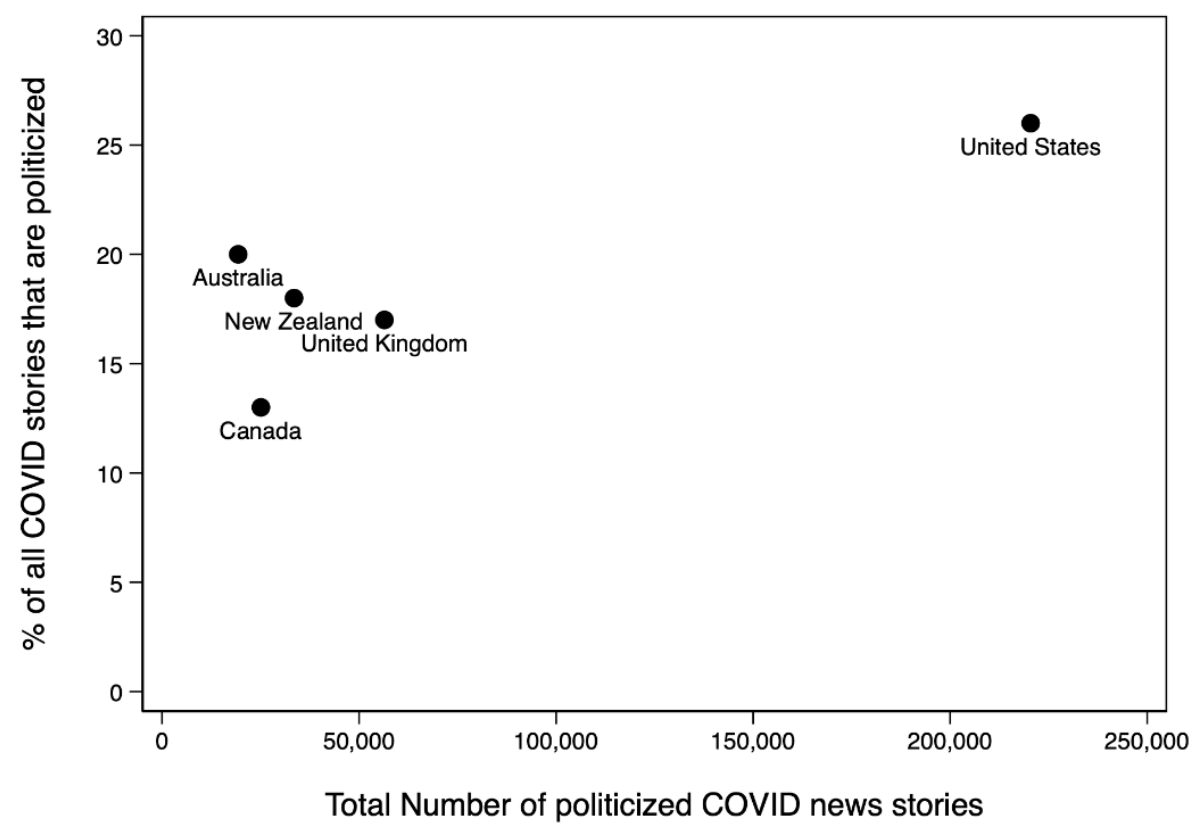

Note: number and percent of COVID-19 media stories between January 1 and July 1, 2020 that were politicized, and each of the five countries.

Our analysis relies on data from the Vox Pop Labs COVID-19 Monitor initiative. Surveys were conducted in the United States, United Kingdom, Canada, Australia, and New Zealand in July of 2020. We have a large, nationally representative sample from each country and the total $N$ is 6,956. To ensure representativeness, we pre-stratified on the basis of age, sex and geography. Post-stratification weights are based on age, education, sex and past vote. In Australia/UK we also used state/region, and in New Zealand we also used Maori identification.

In our analysis, we examine the transmission rates of COVID-19 conspiracies online. Just because an individual sees a conspiracy theory online does not mean they will share it. Therefore, knowing the degree to which the viewing of conspiracies translates 
into sharing them and how that differs across countries is important. We also use a linear model to evaluate the other predictors of sharing that we have identified: trust in social media, trust in health officials, populism, ideology and trust in government. In the linear model, we control for age, gender, education, use of digital news media, and trust in the pharmaceutical industry. This last control is included because one of the conspiracy theories explicitly mentions pharmaceutical industry, and distrust of "Big Pharma" plays a prominent role in vaccine misinformation ${ }^{18}$.

\section{Results}

To begin answering the question 'who is sharing COVID-19 conspiracies?', we first examine the average frequency with which these conspiracies are seen and shared in each country (Figure 2). The frequency with which a respondent views these conspiracies is measured with the question: 'How often do you encounter stories and posts about any of these claims online, for example on social media platforms like Facebook, Twitter, or YouTube?'. The frequency with which a respondent shares these conspiracies is measured with the question: 'Whether or not you agree with them, how often do you yourself share stories and posts about any of these claims online, for example on social media platforms like Facebook, Twitter or YouTube? 'The response scales for both questions is the same: 0-Never to 10-All the time. 
Figure 2. Distribution of Seeing and Sharing COVID-19 Conspiracies by Country

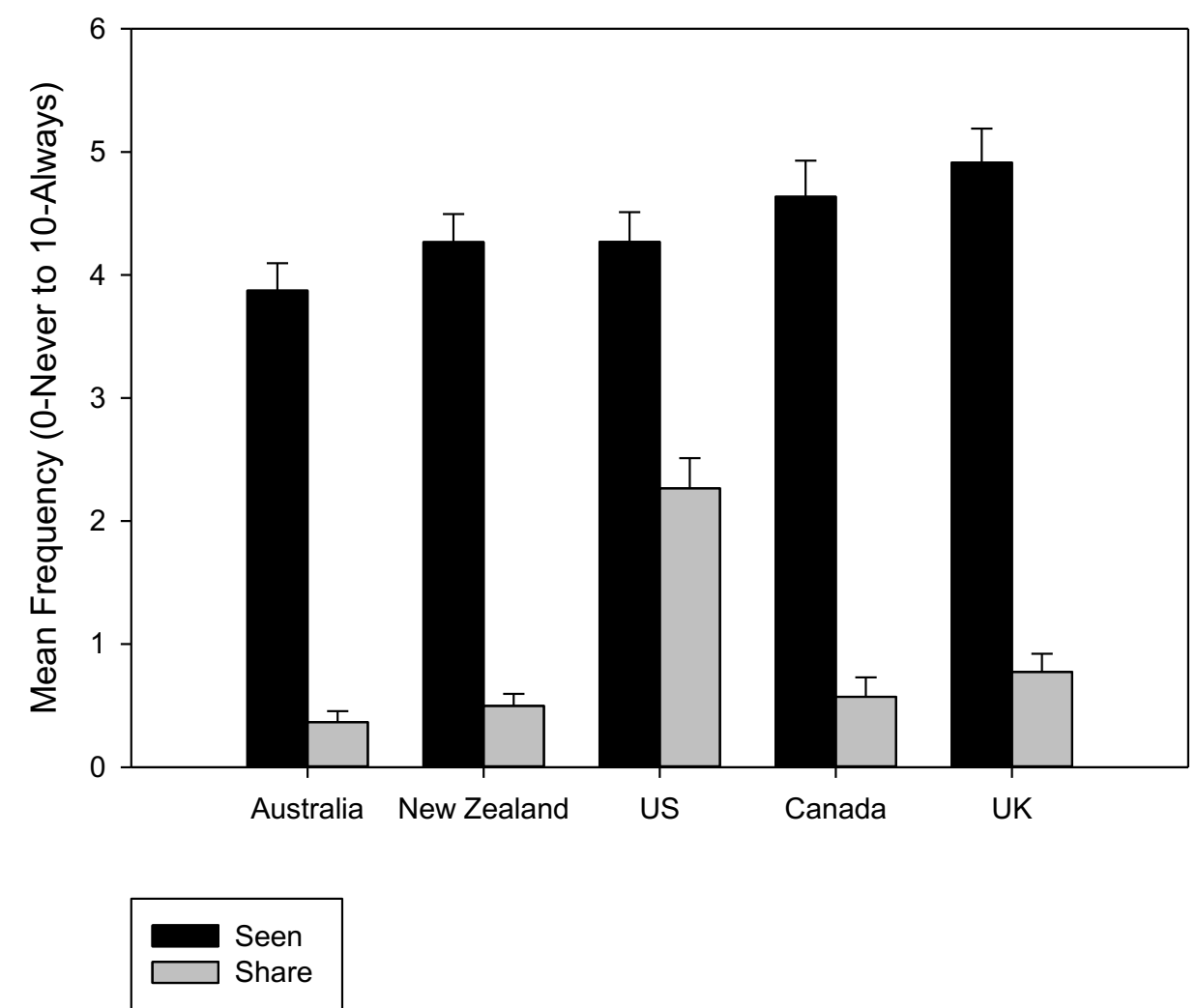

Note: the average frequency (from 0 equals never to 10 equals always) a respondent sees and shares COVID-19 related conspiracies online, in each country (with $95 \%$ confidence interval). $N=1,275$ (US); 1,376 (Canada); 1,107 (UK); 1,976 (Australia); 1,222 (New Zealand).

We see that respondents in Australia report seeing these conspiracies least often, on average, (3.87 on the 0 to 10 scale) and respondents in the UK report seeing these conspiracies most often (4.91). We also can see that there is a correspondence between the average frequency with which the COVID-19 conspiracies are seen and the average frequency with which they are shared, and the frequency with which the conspiracies are shared is a small fraction of the frequency with which they are seen. One country defies this pattern. While the US falls in the middle on the frequency with which conspiracies are seen (4.27), it far surpasses all other countries on the frequency with which conspiracies are shared. In the US, the average frequency with which conspiracies are shared is 2.27 (on the 0 to 10 scale). This is over half the frequency with which they are seen. The next closest nation is the UK with a share frequency of 0.77 . This is less than a fifth of the frequency with which they are seen. 
Figure 3. Transmission of COVID-19 Conspiracies by Country

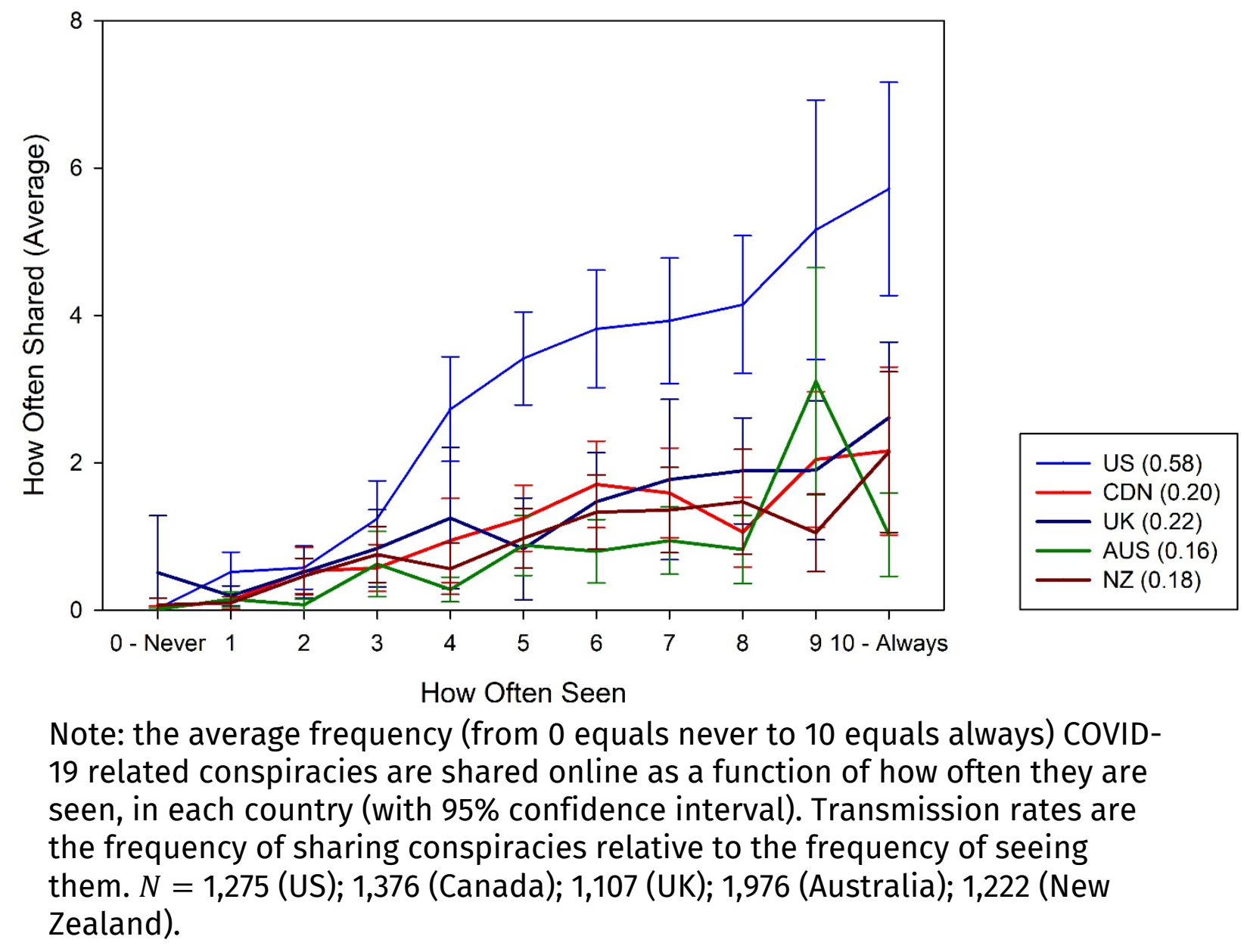

Next, we compute the transmission rate in each country. We calculate the transmission rate by regressing the frequency of conspiracies shared on the frequency of conspiracies seen. Figure 3 reports the transmission rates along with a more detailed look at the relationship between the seeing and sharing of these conspiracies. Consistent with Figure 2, we see that the transmission rate in the US (0.47) is more than three times that of any other country in the study - sharing a conspiracy is about half as frequent as seeing a conspiracy. The next closest is the UK (0.13) and the lowest transmission rate is in Australia (0.09). The figure also shows that as the frequency of seeing these conspiracies increases, so does the frequency of sharing them. This relationship is much stronger in the US compared to other countries. Those that frequently view conspiracies in the US also share them much more often than individuals that frequently view conspiracies in other countries. At the other end of the scale, those that infrequently view conspiracies in the US share them about as often as those that infrequently view conspiracies in other countries. 
So far, the answer to our question 'who shares COVID-19 conspiracies' is: those that often see them, especially Americans. To explore this further, we predict the frequency of sharing conspiracies with our theoretically derived individual level predictors. We do this with a linear regression model, and we include those that have seen a conspiracy, so we are estimating the effect of these predictors conditional on having seen a conspiracy.

In our prediction model we include variables related to the politicization of COVID-19: populism (4 point scale), ideology (11 point scale) and trust that the information about COVID-19 from government is factual and objective ( 4 point scale). We include a variable we expect to be important when COVID-19 is not politicized: trust that the information about COVID-19 from public health officials is factual and objective (4 point scale). We also include similar trust in COVID-19 information from social media (4 point scale) as a predictor of sharing COVID-19 conspiracies. Additionally, we include a number of controls in the model: age, male (vs. female or other), whether the respondent has a university degree, trust in pharmaceutical industry and use of digital sources of news information. This last is measured using the respondent's estimate of how much of their news and information on the COVID-19 pandemic they get from online and social media. This means our estimated effects of trust in social media is controlling for the use of these media platforms.

Figure 4 shows the results of the conspiracy sharing prediction model for each country. Very evident from the results is that trust in social media positively predicts sharing COVID-19 conspiracy theories in all five countries. This effect is strongest in the US, where an increase of 1 (on the 1 to 4 scale) in trust increases the frequency of sharing conspiracies by 1.1 (on the 0 to 10 scale).

The US is distinct in the way that political variables predict the sharing of conspiracies. An increase of 1 (on the 0 to 10 scale) in liberal/conservative scale increases the frequency of sharing conspiracies by 0.4 . An increase of 1 (on the 1 to 4 scale) in populism increases the frequency of sharing conspiracies by 0.9. Ideology does not have an effect in any other country. The only other country where populism predicts conspiracy sharing is in New Zealand, although it does predict conspiracy sharing in the UK with a $P$-value of 0.053 . To the extent that there are political effects on sharing conspiracies, these appear to be captured by ideology and populism as trust in the national/federal government does not have a statistically significant effect (at the 0.05 level) in any country. However, trust in the state government does negatively predict sharing conspiracies in the US with a P-value of 0.058 .

There is weak evidence that in countries in which neither populism nor ideology predict conspiracy sharing, trust in health officials is a predictor. In Canada, an increase of one (on the 1 to 4 scale) in trust in health officials reduces the frequency of sharing conspiracies by $0.4(P$-value $=0.004)$. In Australia, the effect is a reduction of 0.3 but the effect is not statistically significant at the 0.05 level $(P$-value $=0.065)$. 
Figure 4. Predictors of Sharing COVID-19 Conspiracies on Social Media
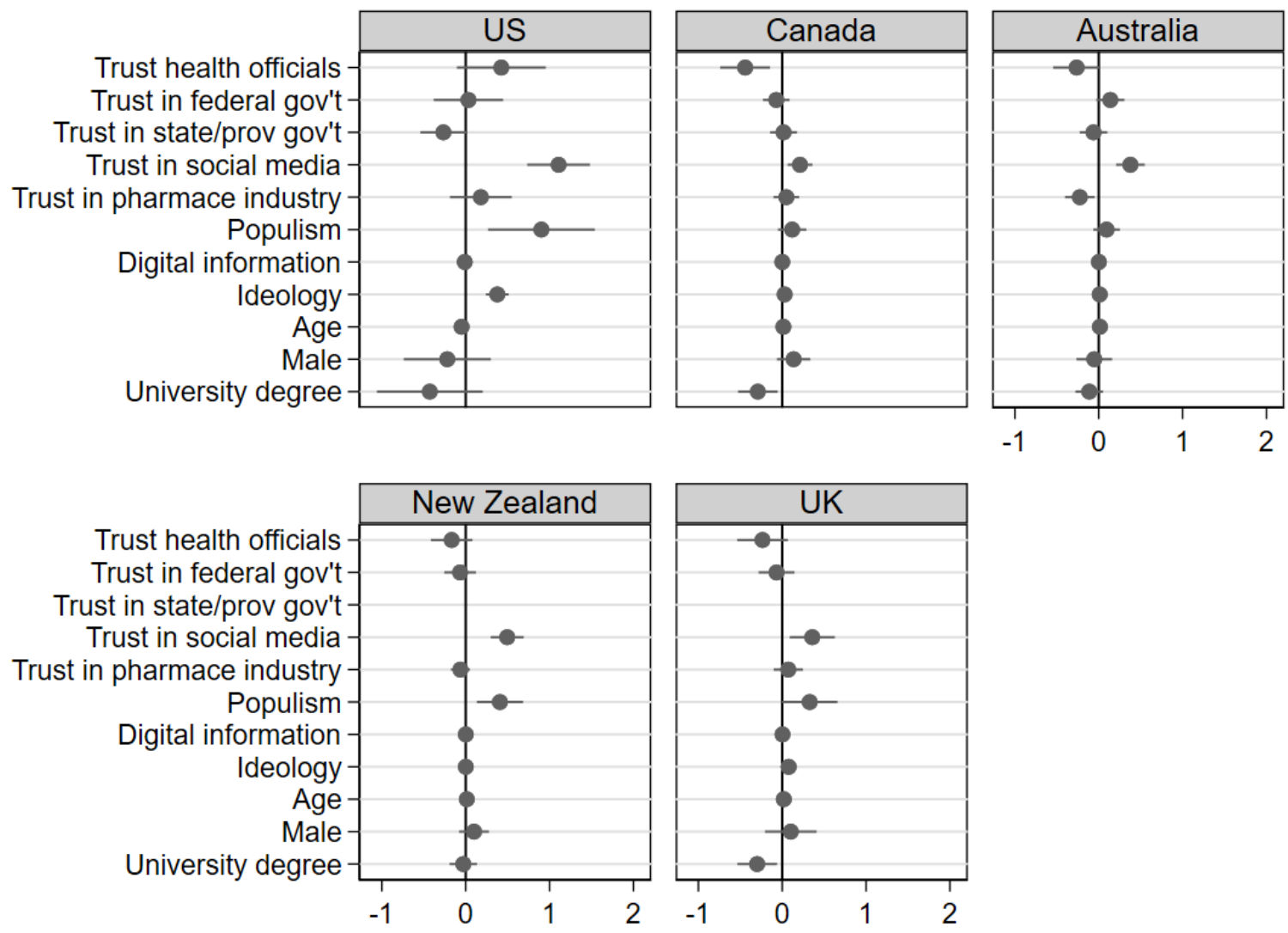

Note: estimated effects from a linear model of hypothesized predictors frequency of of sharing COVID-19 conspiracies on social media (and control variables). $N=$ 743 (US); 1,005 (Canada); 970 (UK); 1,434 (Australia); 743 (New Zealand).

\section{Discussion}

When the entire world stopped in 2020 due to the global COVID-19 pandemic, we were presented with a rare opportunity to study determinants of the same conspiracy beliefs and sharing across multiple countries. Regardless of where one lives, everything changed because of COVID-19, and the conspiracy theories that emerged were largely universal across the world. This presents us with a unique opportunity to study the drivers of these attitudes and behaviors beyond just a single country context. Here, we focused on five peer countries, all Western, English-speaking democracies: the United States, Canada, the United Kingdom, Australia, and New Zealand.

There are common themes between all five countries. Trusting the information on social media about COVID-19 as factual and objective is an important predictor of sharing conspiracies across all five countries in our study. In the age of declining trust in institutions, and scholars thinking of ways to improve trust in the media, elevated trust in social media might actually be an obstacle to reducing misinformation online. 
It seems that skepticism about information on social media should be an important part of any media literacy efforts.

We also find big and important differences between the countries of interest, mostly in the form of US as an outlier. In America, respondents are no more likely to report seeing any of the seven conspiracy theories online than respondents in the other four countries. But they are three times more likely to share these conspiracies with their followers than respondents in the other countries.

Part of this is likely due to the fact that, as was highlighted in Figure 1, COVID-19 pandemic has been politicized in the United States in ways unseen in other countries. This polarized nature of COVID-19 in the US is apparent in the individual level predictors of whether an individual shares COVID-19 conspiracy theories. Ideology is only a significant predictor in the US, and New Zealand is the only other country for which populism is a significant predictor. But populist sentiment is not necessarily stronger in the US than in the other countries. For example, the US populist sentiment has a mean of 2.4. It is the same in the UK, 2.2 in Canada and Australia, and 2.0 in New Zealand. The difference is just that populism is related to sharing conspiracies in the US (and New Zealand). Ideology, on the other hand, is more diverse (more polarized) in the US compared to other nations. Ideology (0 to 10) standard deviations: US, 3.2; Canada, 2.4; UK, 2.4; Australia, 2.3; New Zealand, 2.5. This serves to exacerbate the ideological divide between those that do and do not share conspiracies in the US.

Our findings are consistent with recent work about the dominant role that Americans play in sharing misinformation on social media. A study by Bridgman et al (2021), analyzing a large dataset of Twitter users in Canada, found that the majority of misinformation found on Twitter that is shared by Canadians is retweeted from US accounts. Furthermore, those exposed to American accounts on Twitter were more likely to post misinformation on their accounts. Our findings expand this notion, showcasing the exceptional nature of American conspiracy sharing on social media. Given the global nature of COVID-19 misinformation, this suggests that the sharing behavior of Americans does not simply affect the American context. When Americans flood their social media with conspiracies, it travels abroad and poisons the discourse in other countries.

As governments around the world are trying to vaccinate their populations, containing misinformation and conspiracy theories, particularly those regarding the COVID-19 vaccinations, is of utmost importance. Known anti-vaccine accounts continue to spread on Twitter, Facebook, YouTube, and other platforms, despite official attempts to ban them ${ }^{30}$. We take an important step here in trying to understand the motivations behind the individuals who share misinformation on social media, but more research is needed, especially into what exactly sets the US apart. 


\section{Methods}

News media data source

The data presented in Figure 1 comes from MediaLab Explorer. We used a similar approach to that used in Motta et al (2020). We first searched for a total number stories related to COVID-19 in the national news media of each country between the period of January 1, 2020 and July 1, 2020. The search protocol was: COVID OR coronavirus OR pandemic. We then searched for references to the country's leader and political phrases among these stories. The search protocol was: (COVID OR coronavirus OR pandemic) AND ( *country's leader* OR politics OR political). The leaders were, respectively: Donald Trump in the United States, Justin Trudeau in Canada, Boris Johnson in the United Kingdom, Scott Morrison in Australia, and Jacinda Ardern in New Zealand. MediaLab curates collections of national news sources for each country. In the United States (collection \# 34412234), this includes 271 sources like CNN, ABC News, Fox News, and the New York Times. In Canada (collection \#34411583), this includes 127 sources like the National Post, CBC, and the Toronto Star. In the United Kingdom (collection \#34412476), this includes 97 sources like the BBC, The Independent, and the Guardian. In Australia (collection \#34412282) this includes 62 sources like the Australian Broadcast Company, Sydney Morning Herald, and the Daily Mail Australia. And in New Zealand (collection \#34412098), this includes 82 sources such as the New Zealand Herald, Auckland Now, and Radio New Zealand. All of these collections are dynamic and MediaLab occasionally changes their composition. The searches to collect the data for this paper were conducted on March 14, 2021.

\section{Survey data source}

Respondents to our survey were recruited by Vox Pop Labs for their COVID-19 Monitor initiative in the US (1,275 respondents; July 11- August 13, 2020), Britain (1,107 respondents; July 11-19, 2020), Canada (1,376 respondents; July 11-15, 2020), Australia $(1,976$ respondents; July 10-14, 2020) and New Zealand (1,222 respondents; July 10-17, 2020). The Vox Pop Labs online respondent panel contains more than 1.2 million richlyprofiled panelists from Canada, Australia, New Zealand, the United Kingdom, the United States, France, Germany, and Brazil. To increase representativeness, we prestratified on the basis of age, sex and geography. Post-stratification weights are based on age, education, sex and past vote. In Australia/UK we also used state/region, and in New Zealand we also used Maori identification. 


\section{Survey measures}

We asked respondents about seven prominent COVID-19 related conspiracy theories:

- The Chinese government engineered the coronavirus in a lab.

- The pharmaceutical industry is involved in the spread of the coronavirus.

- There is a link between $5 \mathrm{G}$ technology and the coronavirus.

- The United States military developed the coronavirus as a bioweapon.

- Bill Gates is using the coronavirus to push a vaccine with a microchip capable of tracking people.

- The coronavirus escaped from a lab in Wuhan.

- The worldwide death toll from COVID-19 is highly exaggerated.

We asked respondents how much truth there was to each theory but these measures are not included in this study. Instead, to measure conspiracy theory seeing and sharing online, respondents were asked:

- 'How often do you encounter stories and posts about any of these claims online, for example on social media platforms like Facebook, Twitter, or YouTube?'

- 'How often do you encounter stories and posts about any of these claims online, for example on social media platforms like Facebook, Twitter, or YouTube?'

The response options ranged from never (0) to all the time (10).

We measured trust in social media using responses to a question asking respondents "how much trust do you have in social media to provide factual and objective information about COVID-19?' Response options were: No trust at all (1); Little trust (2); Moderate trust (3); A great deal of trust (4).

Trust in government is measured by using a question that asks respondents "how much trust do you have in the federal government to provide factual and objective information about COVID-19?' Response options were: No trust at all (1); Little trust (2); Moderate trust (3); A great deal of trust (4). Where appropriate, respondents were also asked about their trust in the information from their provincial/state government.

Trust in public health officials is measured using the average response to questions asking respondents "how much trust do you have in: [public health officials, local health care providers, and the World Health Organization (WHO)] to provide factual and objective information about COVID-19?' The response categories are the same as the other trust questions, and the measure is the average response across the three sources.

Populism for each respondent is measure by calculating the average disagreement/agreement across five items: 
- [Country] is divided between ordinary people and the corrupt elites who exploit them.

- The will of the people should be the highest principle in this country's politics.

- It doesn't really matter whom you vote for because the rich control all political parties.

- I'd rather put my trust in the wisdom of ordinary people than the opinions of experts and intellectuals.

- When it comes to really important questions, scientific facts don't help that much.

The response options for each item is the same: Strongly disagree (1); Somewhat disagree (2); Somewhat agree (3); Strongly agree (4); Don't know. This battery is designed to capture two essential aspects of populism: conflict between average citizens and elites, and distrust of experts and intellectuals. It has been used in published research.?

Ideology Is measured using the respondent's self-placement on a left/liberal (0) to right/conservative (10) scale.

Trust in pharmaceutical industry is measured using responses to a question asking respondents how much trust they have in the pharmaceutical industry in general? The response categories are the same as the other trust questions.

Use of digital news is measured by asking respondents what percentage of their news and information on the COVID-19 pandemic they get from online and social media sources?

Finally, age is measured in years, and university degree and male are binary $(0,1)$ demographic variables based on self-reports.

Analysis

The results in Figure 2 were produced by calculating the average response to the frequency of seeing and sharing conspiracy theories online variables and the corresponding 95\% confidence interval in each country. The results in Figure 3 were produced by calculating the average response to the sharing variable separately for individuals grouped by their response to the seeing variable. Again, this was done separately for each country and $95 \%$ confidence intervals were calculated. The reported transmission rates are calculated by regressing frequency of seeing conspiracies on frequency of sharing them in each country. The regression coefficients were estimated by OLS and the resulting slope coefficients are the transmission rates. Using OLS linear regression, the results in Figure 4 were produced by regressing frequency of sharing conspiracy theories online on: trust in social media, trust in public health officials, trust in government, populism, ideology, trust in pharmaceutical 
industry, use of digital news, age, university degree and male. This was done for each country and the samples were restricted to respondents to give an answer of more than ' 0 -Never' to the question about seeing conspiracies online. The results then are estimates of the effects of theorized predictors of sharing conspiracies, given that the respondent had seen at least one of them at least once. 


\section{References}

${ }^{1}$ Enders, A. M., Uscinski, J. E., Klofstad, C. \& Stoler, J. The different forms of COVID-19 misinformation and their consequences. Harvard Kennedy School Misinformation Review (2020) doi:10.37016/mr-2020-48.

${ }^{2}$ Shahsavari, S., Holur, P., Tangherlini, T. R. \& Roychowdhury, V. Conspiracy in the Time of Corona: Automatic detection of COVID-19 Conspiracy Theories in Social Media and the News. arXiv:2004.13783 [cs] (2020).

${ }^{3}$ Motta, M., Stecula, D. \& Farhart, C. How Right-Leaning Media Coverage of COVID-19 Facilitated the Spread of Misinformation in the Early Stages of the Pandemic in the U.S. Canadian Journal of Political Science/Revue canadienne de science politique 53, 335-342 (2020).

${ }^{4}$ Allington, D., Duffy, B., Wessely, S., Dhavan, N. \& Rubin, J. Health-protective behaviour, social media usage and conspiracy belief during the COVID-19 public health emergency. Psychol Med 1-7 doi:10.1017/S003329172000224X.

${ }^{5}$ Bertin, P., Nera, K. \& Delouvée, S. Conspiracy Beliefs, Rejection of Vaccination, and Support for hydroxychloroquine: A Conceptual Replication-Extension in the COVID-19 Pandemic Context. Front. Psychol. 11, (2020).

${ }^{6}$ Roozenbeek, J. et al. Susceptibility to misinformation about COVID-19 around the world. Royal Society Open Science 7, 201199 (2020).

${ }^{7}$ Stecula, D. A. \& Pickup, M. How populism and conservative media fuel conspiracy beliefs about COVID-19 and what it means for COVID-19 behaviors. Research \& Politics 8, 2053168021993979 (2021).

${ }^{8}$ Romer, D. \& Jamieson, K. H. Conspiracy theories as barriers to controlling the spread of COVID-19 in the U.S. Soc Sci Med 263, 113356 (2020).

${ }^{9}$ Lindholt, M. F., Jørgensen, F. J., Bor, A. \& Petersen, M. B. Willingness to Use an Approved COVID-19 Vaccine: Cross-National Evidence on Levels and Individual-Level Predictors. (2020) doi:10.31234/osf.io/8kn5f.

${ }^{10}$ Jolley, D. \& Douglas, K. M. The Effects of Anti-Vaccine Conspiracy Theories on Vaccination Intentions. PLOS ONE 9, e89177 (2014).

${ }^{11}$ Lu, R. \& Sheng, S. Y. From Fear to Hate: How A Pandemic Sparks Racial Animus in the United States. https://papers.ssrn.com/abstract $=3646880$ (2020)

doi:10.2139/ssrn.3646880. 
${ }^{12}$ Papakyriakopoulos, O., Serrano, J. C. M. \& Hegelich, S. The spread of COVID-19 conspiracy theories on social media and the effect of content moderation. Harvard Kennedy School Misinformation Review 1, (2020).

${ }^{13}$ Bridgman, A. et al. The causes and consequences of COVID-19 misperceptions: understanding the role of news and social media. Harvard Kennedy School Misinformation Review 1, (2020).

${ }^{14}$ Jamieson, K. H. \& Albarracín, D. The Relation between Media Consumption and Misinformation at the Outset of the SARS-CoV-2 Pandemic in the US. Harvard Kennedy School Misinformation Review 1, (2020).

${ }^{15}$ Gregory, J. \& McDonald, K. Special Report: COVID-19 Myths. NewsGuard https://www.newsguardtech.com/covid-19-myths/.

${ }^{16}$ Klepper, D., Amiri, F. \& Dupuy, B. The superspreaders behind top COVID-19 conspiracy theories. AP News https://apnews.com/article/conspiracy-theories-iranonly-on-ap-media-misinformation-bfca6d5b236a29d61c4dd38702495ffe (2021).

${ }^{17}$ Van Bavel, J. J. et al. Political Psychology in the Digital (mis)Information age: A Model of News Belief and Sharing. Social Issues and Policy Review 15, 84-113 (2021).

${ }^{18}$ Stecula, D. A., Kuru, O. \& Jamieson, K. H. How trust in experts and media use affect acceptance of common anti-vaccination claims. The Harvard Kennedy School (HKS) Misinformation Review 1, (2020).

${ }^{19}$ Laato, S., Islam, A. K. M. N., Islam, M. N. \& Whelan, E. Why do People Share Misinformation during the COVID-19 Pandemic? European Journal of Information Systems 29, 288-305 (2020).

${ }^{20}$ Douglas, K. M. et al. Understanding Conspiracy Theories. Political Psychology 40, 335 (2019).

${ }^{21}$ Uscinski, J. E. et al. Why do people believe COVID-19 conspiracy theories? Harvard Kennedy School Misinformation Review 1, (2020).

${ }^{22}$ Pennycook, G., McPhetres, J., Bago, B. \& Rand, D. Beliefs about COVID-19 in Canada, the U.K., and the U.S.A.: A novel test of political polarization and motivated reasoning. (2020) doi:10.31234/osf.io/zhjkp.

${ }^{23}$ Pickup, M., Stecula, D. \& Linden, C. van der. Novel Coronavirus, Old Partisanship: COVID-19 Attitudes and Behaviours in the United States and Canada. Canadian Journal of Political Science/Revue canadienne de science politique 53, 357-364 (2020). 
${ }^{24}$ Gadarian, S. K., Goodman, S. W. \& Pepinsky, T. B. Partisanship, health behavior, and policy attitudes in the early stages of the COVID-19 pandemic. PLOS ONE 16, e0249596 (2021).

${ }^{25}$ Clinton, J., Cohen, J., Lapinski, J. \& Trussler, M. Partisan pandemic: How partisanship and public health concerns affect individuals' social mobility during COVID-19. Science Advances 7, eabd7204 (2021).

${ }^{26}$ Guess, A., Nagler, J. \& Tucker, J. Less than you think: Prevalence and predictors of fake news dissemination on Facebook. Science Advances 5, eaau4586 (2019).

${ }^{27}$ Abbasi, K. Covid-19: politicisation, "corruption," and suppression of science. BMJ 371, m4425 (2020).

${ }^{28}$ Green, J., Edgerton, J., Naftel, D., Shoub, K. \& Cranmer, S. J. Elusive consensus:

Polarization in elite communication on the COVID-19 pandemic. Science Advances 6, eabc2717 (2020).

${ }^{29}$ Merkley, E. et al. A Rare Moment of Cross-Partisan Consensus: Elite and Public Response to the COVID-19 Pandemic in Canada. Canadian Journal of Political Science/Revue canadienne de science politique 53, 311-318 (2020).

${ }^{30}$ Ortutay, B. \& Seitz, A. Defying rules, anti-vaccine accounts thrive on social media. AP NEWS https://apnews.com/article/anti-vaccine-accounts-thrive-social-mediae796aaf1ce32d02e215d3b2021a33599 (2021). 